PROCEEDINGS OF THE

AMERICAN MATHEMATICAL SOCIETY

Volume 126, Number 2, February 1998, Pages 607-616

S 0002-9939(98)04066-0

\title{
ACTIONS OF COMPACT QUANTUM GROUPS ON $C^{*}$-ALGEBRAS
}

\author{
MARCIN MARCINIAK
}

(Communicated by Palle E. T. Jorgensen)

\begin{abstract}
In this paper we investigate a structure of the fixed point algebra under an action of compact matrix quantum group on a $C^{*}$-algebra $\mathcal{B}$. We also show that the categories of $\mathcal{C}$-comodules in $\mathcal{B}$ and inner endomorphisms restricted to the fixed point algebra coincide when the relative commutant of the fixed point algebra is trivial. Next we show a version of the Tannaka duality theorem for twisted unitary groups.
\end{abstract}

\section{INTRODUCTION}

In [6] Doplicher and Roberts developed the duality theory for compact groups in the language of abstract symmetric, strict monoidal $C^{*}$-categories. This approach has become a useful tool for description of the superselection structure of a quantum field theory in $(3+1)$-dimensional spacetime by the representation theory of a compact gauge group (see [7]). In order to obtain a similar picture in the lower dimensional spaces ( $c f .[9,10])$ one should consider some class of 'quantum' groups. In $[13,14]$ quasi-Hopf *-algebras have been considered as realizations of quantum gauge groups. However, it is evident that we need a more general class of 'quantum' groups. This statement can be justified by the remark that recently many attempts concerning generalized symmetries of physical systems have been published ( $c f$. Chapter IV in [11], in particular see p. 155). In this paper we shall restrict ourselves to Woronowicz's compact quantum groups ([23]). We are going to investigate the systems composed of a $C^{*}$-algebra $\mathcal{B}$ and a compact quantum group acting on $\mathcal{B}$. It seems to be interesting whether the theory of Doplicher and Roberts can be generalized to the class of such systems.

Our paper is an attempt to describe the duality theory for compact matrix quantum groups. Let us recall that such groups can be considered as quantum Lie groups. So, we will apply the program of $[4,5]$ to quantum $C^{*}$-systems with compact matrix quantum groups and we will obtain similar results. Particularly, the generalized Cuntz algebra associated with the twisted unitary group $S_{q} U(2)$ will be considered. In Corollary 5.7 we give some version of the Tannaka duality theorem for $S_{q} U(2)$. We also show that the fixed point subalgebra has the structure of crossed product of the algebra generated by Jones' projections by a single endomorphism.

Received by the editors May 7, 1996 and, in revised form, July 16, 1996 and August 8, 1996.

1991 Mathematics Subject Classification. Primary 22D25; Secondary 46L60, 81R50.

The author was supported by KBN grant 2 P301 02007.

(C)1998 American Mathematical Society 
The paper is organized as follows: In section 2 we recall some basic definitions concerning compact matrix quantum groups and their representations. In section 3 we prove a kind of duality theorem about equivalences of categories of representations of a quantum group and categories of endomorphisms of a fixed point algebra under an action of the quantum group. In section 4 we establish some basic facts about Cuntz algebras as well as some technical lemmas. In section 5 we study a special example of a quantum $C^{*}$-system composed of the Cuntz algebra and the twisted unitary group.

Part of the paper was done at University of Warsaw. The author wishes to thank Professor S.L. Woronowicz for hospitality and financial support. Special thanks are going to Professor W.A. Majewski for the many helpful discussions during the preparation of the paper.

\section{Compact MATRIX QuANTUM Groups}

Let us recall some basic definitions about compact matrix quantum groups. For details see [23, 24, 25].

Definition 2.1 ([23]). A pair $G=(\mathcal{C}, u)$ is called a compact matrix quantum group (c.m.q.g.) if $\mathcal{C}$ is a unital $C^{*}$-algebra and $u=\left[u_{i j}\right]_{i, j=1, \ldots, N}$ is a matrix with entries in $\mathcal{C}$ such that the following conditions are fulfilled:

1. The ${ }^{*}$-subalgebra $\mathcal{C}_{0}$ of $\mathcal{C}$ generated by entries of $u$ is dense in $\mathcal{C}$.

2. There exists a $C^{*}$-homomorphism (called a comultiplication) $\Delta: \mathcal{C} \rightarrow \mathcal{C} \otimes \mathcal{C}$ such that $\Delta\left(u_{i j}\right)=\sum_{k=1}^{N} u_{i k} \otimes u_{k j}$ for $i, j=1, \ldots, N$.

3. There exists a linear antimultiplicative mapping (coinverse) $\kappa: \mathcal{C}_{0} \rightarrow \mathcal{C}_{0}$ such that $\kappa\left(\kappa\left(C^{*}\right)^{*}\right)=C$ for $C \in \mathcal{C}_{0}$, and $\sum_{k=1}^{N} \kappa\left(u_{i k}\right) u_{k j}=\delta_{i j} I=\sum_{k=1}^{N} u_{i k} \kappa\left(u_{k j}\right)$ for any $i, j=1,2, \ldots, N$. I denotes the unit of the algebra $\mathcal{C}$.

Definition 2.2. Let $G=(\mathcal{C}, u)$ be a c.m.q.g. and $K$ be a finite dimensional Hilbert space. A linear mapping $v: K \rightarrow K \otimes \mathcal{C}$ is called a representation of $G$ (or $\mathcal{C}$-comodule) if $\left(v \otimes \operatorname{id}_{\mathcal{C}}\right) v=\left(\operatorname{id}_{K} \otimes \Delta\right) v$.

Remark 2.3. One can write $v\left(e_{j}\right)=\sum_{i=1}^{d} e_{i} \otimes v_{i j}, j=1, \ldots, d$, where $d=\operatorname{dim} K$, $e_{1}, \ldots, e_{d}$ is an orthonormal basis of $K$, and $v_{i j} \in \mathcal{C}$ for $i, j=1, \ldots, d$. Definition 2.2 yields

$$
\Delta\left(v_{i j}\right)=\sum_{k=1}^{d} v_{i k} \otimes v_{k j}, \quad i, j=1, \ldots, d .
$$

So, given an orthonormal basis of $K$ one has a correspondence between representations of $G$ and elements $v=\left[v_{i j}\right] \in M_{d}(\mathbf{C}) \otimes \mathcal{C}$ satisfying the equation (2.1), where $M_{d}(\mathbf{C})$ stands for the $C^{*}$-algebra of complex $d \times d$ matrices.

The representation $u$ on $\mathbf{C}^{N}$ which in the canonical basis is defined by the matrix $\left[u_{i j}\right]_{i, j=1, \ldots, N}$ is called the fundamental representation of $G$.

Let $v$ and $w$ be representations of $G$ acting on $K$ and $L$ respectively. A linear mapping $T: K \rightarrow L$ is called an intertwining operator between $v$ and $w$ if $w(T e)=$ $\left(T \otimes \operatorname{id}_{\mathcal{C}}\right)(v(e))$ for any $e \in K$. The set of all intertwiners between representations $v$ and $w$ will be denoted by $\operatorname{Mor}(v, w)$. The tensor product of representations $v$ and $w$ is the representation $v \otimes w$ acting on $K \otimes L$ defined by $(v \otimes w)\left(e_{k} \otimes f_{l}\right)=$ $\sum_{i=1}^{d} \sum_{j=1}^{d^{\prime}} e_{i} \otimes f_{j} \otimes v_{i k} w_{j l}, k=1, \ldots, d, l=1, \ldots, d^{\prime}$, where $d=\operatorname{dim} K, d^{\prime}=$ 
$\operatorname{dim} L, e_{1}, \ldots, e_{d}$ and $f_{1}, \ldots, f_{d^{\prime}}$ are orthonormal bases of $K$ and $L$ respectively, and $\left[v_{i k}\right],\left[w_{j l}\right]$ are matrices of $v, w$ in those bases.

A representation $v$ acting on a Hilbert space $K$ is called unitary if the matrix $\left[v_{i j}\right]_{i, j=1, \ldots, d}$ defined in Remark 2.3 is a unitary element of $M_{d}(\mathbf{C}) \otimes \mathcal{C}$, i.e. $\sum_{k=1}^{d} v_{i k} v_{j k}^{*}=\delta_{i j} I=\sum_{k=1}^{d} v_{k i}^{*} v_{k j}$ for any $i, j=1, \ldots, d$.

\section{ACTION OF C.M.Q.G. ON $C^{*}$-ALGEBraS}

Now, let $\mathcal{B}$ be a unital $C^{*}$-algebra. The crucial point in our consideration will be the notion of an action of a c.m.q.g. on the algebra $\mathcal{B}$ (see [8]).

Definition 3.1. Let $G=(\mathcal{C}, u)$ be a c.m.q.g. A morphism $\alpha: \mathcal{B} \rightarrow \mathcal{B} \otimes_{\min } \mathcal{C}$ of $C^{*}$-algebras is called an action of $G$ on $\mathcal{B}$ if $\left(\operatorname{id}_{\mathcal{B}} \otimes \Delta\right) \alpha=\left(\alpha \otimes \operatorname{id}_{\mathcal{C}}\right) \alpha$. The triple $(\mathcal{B}, \alpha, G)$ will be called a quantum $C^{*}$-system over a c.m.q.g. $G$.

A Hilbert space in a $C^{*}$-algebra $\mathcal{B}$ is a finite dimensional closed linear subspace $H$ of $\mathcal{B}$ such that $\psi^{*} \psi^{\prime} \in \mathbf{C} I$ for all $\psi, \psi^{\prime} \in H$ (cf. [5]). The scalar product $(\cdot, \cdot)$ on the Hilbert space $H$ is defined by $\left(\psi, \psi^{\prime}\right) I=\psi^{*} \psi^{\prime}$. So, for an orthonormal basis $\psi_{1}, \ldots, \psi_{d}$ of $H$ one has $\psi_{i}^{*} \psi_{j}=\delta_{i j} I, i, j=1, \ldots, d$. Support of $H$ is the projection $1_{H}=\sum_{i=1}^{d} \psi_{i} \psi_{i}^{*}$, and it does not depend on the choice of orthonormal basis in $H$. Moreover, one can define the inner endomorphism of $\mathcal{B}$ associated with $H: \rho_{H}(B)=\sum_{i=1}^{d} \psi_{i} B \psi_{i}^{*}$ for $B \in \mathcal{B}$, where $\psi_{1}, \ldots, \psi_{d}$ is any orthonormal basis of $H$. Clearly,

$$
1_{H}=\rho_{H}(I) \quad \text { and } \quad \psi B=\rho_{H}(B) \psi, \quad \psi \in H, B \in \mathcal{B} .
$$

The tensor product of two Hilbert spaces $H, H^{\prime}$ in $\mathcal{B}$ is the Hilbert space $H H^{\prime}=$ $\operatorname{span}\left\{\psi \psi^{\prime}: \psi \in H, \psi^{\prime} \in H^{\prime}\right\}$. Observe that $\rho_{H H^{\prime}}=\rho_{H} \rho_{H^{\prime}}$.

Definition 3.2. Let $(\mathcal{B}, \alpha, G)$ be a quantum $C^{*}$-system. We say that a Hilbert space $H$ in $\mathcal{B}$ is a Hilbert $\mathcal{C}$-comodule in $(\mathcal{B}, \alpha, G)$ if $\alpha(H) \subset H \otimes \mathcal{C}$ and the representation $\alpha_{H}$ of $G$ obtained by the restriction of the action $\alpha$ to $H$ is the unitary one.

Lemma 3.3. Let $H$ be a Hilbert $\mathcal{C}$-comodule in $(\mathcal{B}, \alpha, G)$. Then $\alpha\left(1_{H}\right)=1_{H} \otimes I$.

Proof. Let $\left[v_{i j}\right]_{i, j=1, \ldots, d}$ be a unitary matrix of the representation $\alpha_{H}$ in an orthonormal basis $\psi_{1}, \ldots, \psi_{d}$ of $H$ (cf. Remark 2.3). Then $\alpha\left(1_{H}\right)=\sum_{i} \alpha\left(\psi_{i}\right) \alpha\left(\psi_{i}^{*}\right)=$ $\sum_{i} \sum_{k, l} \psi_{k} \psi_{l}^{*} \otimes v_{k i} v_{l i}^{*}=\sum_{k, l} \psi_{k} \psi_{l}^{*} \otimes \sum_{i} v_{k i} v_{l i}^{*}=\sum_{k} \psi_{k} \psi_{k}^{*} \otimes I=1_{H} \otimes I$.

By $\mathcal{U}(\mathcal{B}, \alpha, G)$ we will denote a category of Hilbert $\mathcal{C}$-comodules in $(\mathcal{B}, \alpha, G)$. More precisely, objects of $\mathcal{U}(\mathcal{B}, \alpha, G)$ are Hilbert $\mathcal{C}$-comodules $H$ in $(\mathcal{B}, \alpha, G)$ and morphisms between two comodules $H$ and $H^{\prime}$ are intertwiners between representations $\alpha_{H}$ and $\alpha_{H^{\prime}}$, i.e.

$$
\operatorname{Mor}_{\mathcal{U}}\left(H, H^{\prime}\right)=\left\{\begin{array}{cc}
T 1_{H}=T=1_{H^{\prime}} T, \quad T H \subset H^{\prime}, \\
\alpha_{H^{\prime}}(T \psi)=(T \otimes I) \alpha_{H}(\psi), \psi \in H
\end{array}\right\} .
$$

Let $\mathcal{B}^{\alpha}$ denote the subalgebra of fixed points under the action $\alpha$, i.e. $\mathcal{B}^{\alpha}=\{A \in$ $\mathcal{B}: \alpha(A)=A \otimes I\}$.

Proposition 3.4. Suppose $H, H^{\prime}$ are Hilbert $\mathcal{C}$-comodules in $(\mathcal{B}, \alpha, G)$, and $T \in$ $\operatorname{Mor}_{\mathcal{U}}\left(H, H^{\prime}\right)$. Then $T \in \mathcal{B}^{\alpha}$.

Proof. Lemma 3.3 implies that $\alpha(T)=\alpha\left(T 1_{H}\right)=\sum_{i} \alpha_{H^{\prime}}\left(T \psi_{i}\right) \alpha\left(\psi_{i}^{*}\right)=$ $\sum_{i}(T \otimes I) \alpha_{H}\left(\psi_{i}\right) \alpha\left(\psi_{i}^{*}\right)=(T \otimes I) \alpha\left(1_{H}\right)=T \otimes I$. 
Lemma 3.5. Let $H$ be an object in $\mathcal{U}(\mathcal{B}, \alpha, G)$. Then $\rho_{H}\left(\mathcal{B}^{\alpha}\right) \subset \mathcal{B}^{\alpha}$.

Proof. Let $\psi_{1}, \ldots, \psi_{d}$ and $\left[v_{i j}\right]_{i, j=1, \ldots, d}$ be as in the proof of Lemma 3.3 and $A \in \mathcal{B}^{\alpha}$. Then $\alpha\left(\rho_{H}(A)\right)=\sum_{i} \alpha\left(\psi_{i}\right) \alpha(A) \alpha\left(\psi_{i}\right)^{*}=\sum_{i, j, k} \psi_{j} A \psi_{k}^{*} \otimes v_{j i} v_{k i}^{*}=$ $\sum_{j} \psi_{j} A \psi_{j}^{*} \otimes I=\rho_{H}(A) \otimes I$.

Let $\sigma_{H}$ be an endomorphism of $\mathcal{B}^{\alpha}$ obtained by the restriction $\rho_{H}$ to $\mathcal{B}^{\alpha}$. By $\mathcal{I}(\mathcal{B}, \alpha, G)$ we denote the category such that its objects are endomorphisms $\sigma_{H}$ with $H$ being an object in $\mathcal{U}(\mathcal{B}, \alpha, G)$ while morphisms of $\mathcal{I}(\mathcal{B}, \alpha, G)$ are defined as follows: for any two endomorphisms $\sigma, \sigma^{\prime}$

$$
\operatorname{Mor}_{\mathcal{I}}\left(\sigma, \sigma^{\prime}\right)=\left\{T \in \mathcal{B}^{\alpha}: T \sigma(I)=T=\sigma^{\prime}(I) T, T \sigma(A)=\sigma^{\prime}(A) T, A \in \mathcal{B}^{\alpha}\right\} .
$$

Both $\mathcal{U}(\mathcal{B}, \alpha, G)$ and $\mathcal{I}(\mathcal{B}, \alpha, G)$ have the structure of monoidal $C^{*}$-categories ( $c f$. $[5,6])$.

Theorem 3.6. Let $(\mathcal{B}, \alpha, G)$ be a quantum $C^{*}$-system over a c.m.q.g. $(\mathcal{C}, u)$. Then there is a faithful functor $F$ from $\mathcal{U}(\mathcal{B}, \alpha, G)$ to $\mathcal{I}(\mathcal{B}, \alpha, G)$. If also $\left(\mathcal{B}^{\alpha}\right)^{\prime} \cap \mathcal{B}=C I$, then $F$ is an isomorphism of monoidal $C^{*}$-categories.

Proof. Define $F$ on an object $H$ of $\mathcal{U}(\mathcal{B}, \alpha, G)$ as $F(H)=\sigma_{H}$ and suppose that $T \in \operatorname{Mor}_{\mathcal{U}}\left(H, H^{\prime}\right)$ for some objects $H, H^{\prime}$ of $\mathcal{U}(\mathcal{B}, \alpha, G)$. By Proposition $3.4 T \in$ $\mathcal{B}^{\alpha}$, while (3.2) implies $T \sigma_{H}(I)=T=\sigma_{H^{\prime}}(I) T$. (3.2) gives also $T \sigma_{H}(A) \psi=$ $T \psi A=\sigma_{H^{\prime}}(A) T \psi$, for $A \in \mathcal{B}^{\alpha}, \psi \in H$. So, $T \sigma_{H}(A) 1_{H}=\sigma_{H^{\prime}}(A) T 1_{H}$, hence $T \in$ $\operatorname{Mor}_{\mathcal{I}}\left(\sigma_{H}, \sigma_{H^{\prime}}\right)$. Then, we can define $F(T)=T$ and $F$ is a faithful functor. Given $\phi \in \mathcal{B}$ such that $\phi A=\sigma_{H}(A) \phi$ for $A \in \mathcal{B}^{\alpha}$, one has $\psi^{*} \phi A=\psi^{*} \sigma_{H}(A) \phi=A \psi^{*} \phi$ for $\psi \in H$. So, $\psi^{*} \phi \in\left(\mathcal{B}^{\alpha}\right)^{\prime} \cap \mathcal{B}$. Assuming $\left(\mathcal{B}^{\alpha}\right)^{\prime} \cap \mathcal{B}=\mathbf{C} I$ one obtain $\psi_{i}^{*} \phi=\lambda_{i} I$ for an orthonormal basis $\psi_{1}, \ldots, \psi_{d}$ of $H$. Hence, $\phi=\sigma_{H}(I) \phi=\sum_{i} \psi_{i} \psi_{i}^{*} \phi=\sum_{i} \lambda_{i} \psi_{i} \in$ $H$. So, the Hilbert space $H$ is uniquely determined by its inner endomorphism $\sigma_{H}$, i.e. $H=\left\{\phi \in \mathcal{B}: \phi A=\sigma_{H}(A) \phi\right.$ for $\left.A \in \mathcal{B}^{\alpha}\right\}$. Let $T \in \operatorname{Mor}_{\mathcal{I}}\left(\sigma_{H}, \sigma_{H^{\prime}}\right)$ and let $\psi_{1}, \ldots, \psi_{d}\left(\psi_{1}^{\prime}, \ldots, \psi_{d^{\prime}}^{\prime}\right)$ be a basis of $H$ ( $H^{\prime}$ respectively). Then, $\psi_{j}{ }^{\prime *} T \psi_{i} A=$ $\psi_{j}{ }^{\prime *} T \sigma_{H}(A) \psi_{i}={\psi_{j}}^{\prime *} \sigma_{H^{\prime}}(A) T \psi_{i}=A \psi_{j}{ }^{\prime *} T \psi_{i}, A \in \mathcal{B}^{\alpha}$. Thus ${\psi_{j}}^{{ }^{*}} T \psi_{i}=\lambda_{j i} I$ for $i=1, \ldots, d, j=1, \ldots, d^{\prime}$. So $T=\sigma_{H^{\prime}}(I) T \sigma_{H}(I)=\sum_{i, j} \lambda_{j i} \psi_{j}{ }^{\prime} \psi_{i}^{*}$, hence $T H \subset H^{\prime}$. Moreover, $(T \otimes I) \alpha(\psi)=\alpha(T) \alpha(\psi)=\alpha(T \psi)$ for $\psi \in H$. Consequently, we conclude that $T \in \operatorname{Mor}_{\mathcal{U}}\left(H, H^{\prime}\right)$.

\section{Preliminaries about Cuntz algebras}

To describe the action of the quantum group $S_{q} U(2)$ we will need some basic facts about Cuntz algebras as well as some technical lemmas. Therefore, for the reader's convenience we will provide the necessary material in this section.

Recall that the Cuntz algebra $\mathcal{O}_{d}$ for $d \in \mathbf{N}, d>1$, is a $C^{*}$-algebra generated by $d$ isometries $\psi_{1}, \ldots, \psi_{d}$ such that $\psi_{i}^{*} \psi_{j}=\delta_{i j} I, i, j=1, \ldots, d$, and $\sum_{i=1}^{d} \psi_{i} \psi_{i}^{*}=I$. In [3] Cuntz has showed that $\mathcal{O}_{d}$ is uniquely defined by these conditions and it is simple, i.e. it contains no nontrivial ideals.

Let $H=\operatorname{span}\left\{\psi_{1}, \ldots, \psi_{d}\right\}$, and let $H^{k}$ denote the $k$-th tensor power of $H$, i.e. $H^{k}=\operatorname{span}\left\{\psi_{i_{1}} \ldots \psi_{i_{k}}: i_{1}, \ldots, i_{k}=1, \ldots, d\right\}$. Linear operators from $H^{k}$ to $H^{l}$ can be identified with elements of $H^{l} H^{* k}$, where $H^{l} H^{* k}=\operatorname{span}\left\{\phi_{1} \phi_{2}^{*}: \phi_{1} \in\right.$ $\left.H^{l}, \phi_{2} \in H^{k}\right\}$. Let us observe that for every $k, l \geq 0, \phi_{1} \in H^{l}, \phi_{2} \in H^{k}$ we can write $\phi_{1} \phi_{2}^{*}=\sum_{i=1}^{d} \phi_{1} \psi_{i} \psi_{i}^{*} \phi_{2}^{*} \in H^{l+1} H^{*(k+1)}$. Hence, there is the natural inclusion $H^{l} H^{* k} \subset H^{l+1} H^{*(k+1)}$. 
By $\mathcal{P}_{d}$ we will denote the dense ${ }^{*}$-subalgebra in $\mathcal{O}_{d}$ generated algebraically by $\psi_{1}, \ldots, \psi_{d}$. The structure of $\mathcal{P}_{d}$ can be described by saying that it is a graded ${ }^{*}$-algebra, i.e. $\mathcal{P}_{d}=\bigoplus_{k \in \mathbf{Z}} \mathcal{P}_{d}^{k}$, where

$$
\mathcal{P}_{d}^{k}=\bigcup_{n, n+k \geq 0} H^{n+k} H^{* n}
$$

In order to get the similar description of $\mathcal{O}_{d}$ we will consider the gauge action $\beta$ of the one dimensional torus $T^{1}$ on $\mathcal{O}_{d}$ which is determined by the condition: $\beta_{t}\left(\psi_{i}\right)=t \psi_{i}$ for $t \in T^{1}, i=1, \ldots, d$. Let $m_{k}(X)=\int_{T^{1}} t^{-k} \beta_{t}(X) d t$ for each $k \in \mathbf{Z}$ and $X \in \mathcal{O}_{d}$, where $d t$ stands for the normalized Haar measure on the group $T^{1}$. Then $m_{k}$ is a projection of norm 1 defined on the Banach space $\mathcal{O}_{d}$. Let $\mathcal{O}_{d}^{k}$ be the range of $m_{k}$ i.e. $\mathcal{O}_{d}^{k}=\left\{X \in \mathcal{O}_{d}: m_{k}(X)=X\right\}$. Then we have easy

Proposition 4.1. Let $k \in Z$. Then

i) $\mathcal{O}_{d}^{k}=\left\{X \in \mathcal{O}_{d}: \beta_{t}(X)=t^{k} X, t \in T^{1}\right\}$,

ii) $\mathcal{O}_{d}^{k}=\overline{\mathcal{P}_{d}^{k}}$.

The projection $m_{0}$ is in fact a completely positive conditional expectation onto the fixed point subalgebra $\mathcal{O}_{d}^{0}$. Moreover, for every $X \in \mathcal{P}_{d}$ we have $X=\sum_{k} m_{k}(x)$.

Let us remark that the fixed point subalgebra $\mathcal{O}_{d}^{0}$ is the inductive limit of matrix algebras $(c f .(4.1))$, so it has a structure of UHF-algebra of type $d^{\infty}$.

Proposition 4.2. For $X \in \mathcal{O}_{d}$, if $m_{k}(X)=0$ for each $k \in Z$ then $X=0$.

Proof. Use a similar argument to that given in the proof of Proposition 1.10 in $[3]$.

Let $G=(\mathcal{C}, u)$ be a c.m.q.g. Given a unitary representation of $G$ on $H$ defined in the basis $\psi_{1}, \ldots, \psi_{d}$ by a matrix $v=\left[v_{i j}\right]_{i, j=1, \ldots, d}$ we can determine an action $\alpha$ of $G$ on $\mathcal{O}_{d}$ by defining it on generators (see [12], [8]):

$$
\alpha\left(\psi_{j}\right)=\sum_{i=1}^{d} \psi_{i} \otimes v_{i j}, \quad j=1, \ldots, d .
$$

Definition (4.2) leads to $\alpha\left(H^{k} H^{* k}\right) \subset H^{k} H^{* k} \otimes \mathcal{C}$ for every $k=1,2, \ldots$, i.e. $H^{k} H^{* k}$ is invariant with respect to the action $\alpha$ and so is $\mathcal{O}_{d}^{0}$.

Further, let us recall that the Haar measure $h$ on $G$ can be considered as a state on $\mathcal{C}$ such that $\left(h \otimes \operatorname{id}_{\mathcal{C}}\right) \Delta(a)=h(a) I=\left(\operatorname{id}_{\mathcal{C}} \otimes h\right) \Delta(a)$, for any $a \in \mathcal{C}$. It is known $([23])$ that each c.m.q.g admits the unique Haar measure. Let us define $E_{\alpha}=\left(\operatorname{id}_{\mathcal{O}_{d}} \otimes h\right) \alpha$. Then $E_{\alpha}$ is a projection of norm 1 from $\mathcal{O}_{d}$ onto the fixed point algebra $\mathcal{O}_{d}^{\alpha}$ under the action $\alpha([12])$. It is easy to check that the action $\alpha$ of c.m.q.g. $G$ commutes with the action $\beta$ of $T^{1}$, i.e. $\alpha\left(\beta_{t}(X)\right)=\left(\beta_{t} \otimes \mathrm{id}_{\mathcal{C}}\right) \alpha(X)$, for every $X \in \mathcal{O}_{d}$ and $t \in T^{1}$.

Proposition 4.3. If $X \in \mathcal{O}_{d}^{\alpha}$ then $m_{k}(X) \in \mathcal{O}_{d}^{\alpha}$ for each $k \in \boldsymbol{Z}$.

Proof. Let $X \in \mathcal{O}_{d}^{\alpha}$. Then

$$
\begin{aligned}
\alpha\left(m_{k}(X)\right) & =\int_{T^{1}} t^{-k} \alpha\left(\beta_{t}(X)\right) d t=\int_{T^{1}} t^{-k}\left(\beta_{t} \otimes \operatorname{id}_{\mathcal{C}}\right)(\alpha(X)) d t \\
& =\int_{T^{1}} t^{-k}\left(\beta_{t} \otimes \operatorname{id}_{\mathcal{C}}\right)(X \otimes I) d t=\int_{T^{1}} t^{-k}\left(\beta_{t}(X) \otimes I\right) d t \\
& =\int_{T^{1}} t^{-k} \beta_{t}(X) d t \otimes I=m_{k}(X) \otimes I .
\end{aligned}
$$


Proposition 4.4. For every $k \in \boldsymbol{Z}$ one has $E_{\alpha}\left(\mathcal{O}_{d}^{k}\right) \subset \mathcal{O}_{d}^{k}$ and $E_{\alpha}\left(\mathcal{P}_{d}^{k}\right) \subset \mathcal{P}_{d}^{k}$.

Proof. Let $X \in \mathcal{O}_{d}^{k}$, i.e. $\beta_{t}(X)=t^{k} X$. Then

$$
\begin{aligned}
\beta_{t}\left(E_{\alpha}(X)\right) & =\left(\beta_{t} \otimes \operatorname{id}_{\mathbf{C}}\right)\left(\operatorname{id}_{\mathcal{O}_{d}} \otimes h\right) \alpha(X)=\left(\operatorname{id}_{\mathcal{O}_{d}} \otimes h\right)\left(\beta_{t} \otimes \operatorname{id}_{\mathcal{C}}\right) \alpha(X) \\
& =\left(\operatorname{id}_{\mathcal{O}_{d}} \otimes h\right) \alpha\left(\beta_{t}(X)\right)=t^{k} E_{\alpha}(X) .
\end{aligned}
$$

Hence $X \in \mathcal{O}_{d}^{k}$. The second inclusion is implied by the obvious one $E_{\alpha}\left(\mathcal{P}_{d}\right) \subset$ $\mathcal{P}_{d}$.

Let $\alpha^{n}$ denote the restriction of $\alpha$ to the space $H^{n}$. Our notation follows from the observation that such a restriction can be considered as the $n$-th tensor power of the representation $v(c f$. the definition of $\alpha$ in (4.2)). We shall need the category $\mathcal{U}$ of tensor powers of the representation $\alpha$, i.e. $\operatorname{Obj} \mathcal{U}=\left\{\alpha^{n}: n=0,1,2, \ldots\right\}$ (where $\left.\alpha^{0}: \mathbf{C} I \rightarrow \mathbf{C} I \otimes \mathcal{C}: I \rightarrow I \otimes I\right)$ and $\operatorname{Mor}\left(\alpha^{n}, \alpha^{m}\right)$ are the intertwining elements. Let us remark that $\mathcal{U}$ is a monoidal $C^{*}$-category. By Proposition 3.4 we know that morphisms of the category $\mathcal{U}$ are in the fixed point algebra $\mathcal{O}_{d}^{\alpha}$.

Let $\rho(X)=\sum_{i} \psi_{i} X \psi_{i}^{*}, X \in \mathcal{O}_{d}$, be the canonical inner endomorphism of $\mathcal{O}_{d}$ and $\sigma$ be its restriction to the subalgebra $\mathcal{O}_{d}^{\alpha}$. We define a category $\mathcal{I}$ with $\operatorname{Obj} \mathcal{I}=$ $\left\{\sigma^{n}: n=0,1,2, \ldots\right\}$ and $\operatorname{Mor}\left(\sigma^{n}, \sigma^{m}\right)=\left\{T \in \mathcal{O}_{d}^{\alpha}: T \sigma^{n}(A)=\sigma^{m}(A) T, A \in \mathcal{O}_{d}^{\alpha}\right\}$. As in the proof of Theorem 3.6 one can see that $\operatorname{Mor}\left(\alpha^{n}, \alpha^{m}\right) \subset \operatorname{Mor}\left(\sigma^{n}, \sigma^{m}\right)$. The converse inclusion will be showed in the special case when $G$ is exactly the $S_{q} U(2)$.

We conclude this section with the following two lemmas.

Lemma 4.5. Let $p \in N$ and $S \in H^{p}$ be an isometry, i.e. $S^{*} S=I$. If $X \in \mathcal{O}_{d}^{0}$ commutes with $S$ or with $S^{*}$ then $X \in C I$.

Proof. The assumption of the lemma implies $X=S^{* n} X S^{n}$ for every $n \in \mathbf{N}$. On the other hand, let us observe that for every $r \in \mathbf{N}$ we can choose $n_{r} \in \mathbf{N}$ and $X_{r} \in H^{p n_{r}} H^{* p n_{r}}$ such that $\left\|X-X_{r}\right\|<r^{-1}$. The conditions $S^{* n_{r}} \in H^{* p n_{r}}$ and $S^{n_{r}} \in H^{p n_{r}}$, for each $r$, imply: there exist $\lambda_{r} \in \mathbf{C}$ such that $S^{* n_{r}} X_{r} S^{n_{r}}=\lambda_{r} I$. Consequently, we have

$$
\begin{aligned}
\left\|X-\lambda_{r} I\right\| & =\left\|S^{* n_{r}} X S^{n_{r}}-S^{* n_{r}} X_{r} S^{n_{r}}\right\|=\left\|S^{* n_{r}}\left(X-X_{r}\right) S^{n_{r}}\right\| \\
& \leq\left\|X-X_{r}\right\|<r^{-1} .
\end{aligned}
$$

Hence, $\lim _{r \rightarrow \infty} \lambda_{r} I=X$, and $X \in \mathbf{C} I$.

Lemma 4.6. Let $p \in N$ and $S \in H^{p}$ be an isometry. If $X \in \mathcal{O}_{d}$ commutes with $S$ and $S^{*}$ then $X \in C I$.

Proof. Let $X \in \mathcal{O}_{d}$. Then, $m_{l}(X S)=\int t^{-l} \beta_{t}(X S) d t=\int t^{-l} \beta_{t}(X) t^{p} S d t=$ $m_{l-p}(X) S$ for $l \in \mathbf{Z}$. In the same way we can check that $m_{l}(S X)=S m_{l-p}(X)$. Hence, if $X$ commutes with $S$ then $m_{k}(X)$ commutes with $S$ for every $k \in \mathbf{Z}$. Similarly, if $X$ commutes with $S^{*}$ then $m_{k}(X)$ commutes with $S^{*}$. So (cf. Proposition 4.1), for each $k \in \mathbf{Z}$ elements $m_{k}(X)^{*} m_{k}(X), m_{k}(X) m_{k}(X)^{*}$ of $\mathcal{O}_{d}^{0}$ commute with $S$ and by preceding lemma they are multiples of unity. Hence, $m_{k}(X)$ is a multiple of unitary element $U \in \mathcal{O}_{d}$. Using the argument given in [2] (see the proof of Theorem 3.2, see also [1]) one can show that if there is a unitary $U$ in $\mathcal{O}_{d}^{k}$ then $k=0$. Namely, taking $U^{*}$ instead $U$ if necessary, we can assume that $k \geq 0$. Then, $V=U \psi_{1}^{* k} \in \mathcal{O}_{d}^{0}, V V^{*}=I$ and $V^{*} V=\psi_{1}^{k} \psi_{1}^{* k}$, where $\psi_{1}$ is one of the generators of $\mathcal{O}_{d}$. If $\omega$ is the trace on the UHF-algebra $\mathcal{O}_{d}^{0}$ then 
$d^{-k}=\omega\left(\psi_{1}^{k} \psi_{1}^{* k}\right)=\omega\left(V^{*} V\right)=\omega\left(V V^{*}\right)=\omega(I)=1$. Hence $k=0$. It follows that $m_{k}(X)=0$ unless $k=0$, and $m_{0}(X) \in \mathbf{C} I$ by Lemma 4.5. Hence, by Proposition $4.2 X \in \mathbf{C} I$.

\section{Action of $S_{q} U(2)$}

Recall ([22]) that $S_{q} U(2)$ is the pair $(\mathcal{A}, u)$ where $\mathcal{A}$ is the $C^{*}$-algebra generated by two elements $a, c$ fulfilling the following relations:

$$
\begin{aligned}
& a^{*} a+c^{*} c=I, \quad a a^{*}+q^{2} c c^{*}=I, \\
& c c^{*}=c^{*} c, \quad q c a=a c, \quad q c^{*} a=a c^{*},
\end{aligned}
$$

where $q \in[-1,1], q \neq 0$, and $u$ is the matrix

$$
u=\left(\begin{array}{cc}
a & -q c^{*} \\
c & a^{*}
\end{array}\right) .
$$

Now, we want to describe the structure of the fixed point algebra $\mathcal{O}_{2}^{\alpha}$. For this we need some lemma about the Haar measure on $S_{q} U(2)$. For $k \in \mathbf{Z}, n, m \in \mathbf{N}_{0}$ $\left(\mathbf{N}_{0}=\mathbf{N} \cup\{0\}\right)$ we define $a_{k n m}=a^{k} c^{n} c^{* m}$ where for negative $k$ we set $a^{k}=a^{*|k|}$. Then it is known ([22], Theorem 1.2) that the set $\left\{a_{k n m}: k \in \mathbf{Z}, n, m \in \mathbf{N}_{0}\right\}$ is the linear basis of the ${ }^{*}$-algebra $\mathcal{A}_{0}$ generated by elements $a, c$. Hence, for every $b \in \mathcal{A}_{0}$ one has $b=\sum_{k, n, m} \lambda_{k n m} a_{k n m}$, where coefficients $\lambda_{k n m}$ are uniquely defined and $\lambda_{k n m}=0$ for almost every system $(k, n, m)$.

Lemma 5.1. Let $r \in N$ and let $b_{1}, b_{2}, \ldots, b_{r} \in\left\{a, a^{*}, c, c^{*}\right\}$. If $b_{1} b_{2} \ldots b_{r}=$ $\sum \lambda_{k n m} a_{k n m}$ then for every $k, n, m$ we have that $\lambda_{k n m} \neq 0$ implies $k+n+m \equiv$ $r(\bmod 2)$.

Proof. Induction with respect to $r$. If $r=1$ then the statement is obvious. Suppose that it is true for some $r$ and let $b_{1}, \ldots, b_{r}, b_{r+1}$ be as in the lemma. Then $b_{1} \ldots b_{r} b_{r+1}=\sum \lambda_{k n m} a_{k n m} b_{r+1}$ and by the inductive assumption, if $\lambda_{k n m} \neq 0$ then $k+n+m-r$ is even. Relations (5.1) and simple calculations lead to: for every $k, n, m$ one has $a_{k n m} b_{r+1}=\sum_{k^{\prime}, n^{\prime}, m^{\prime}} \lambda_{k^{\prime} n^{\prime} m^{\prime}}^{k n m} a_{k^{\prime} n^{\prime} m^{\prime}}$, where $\lambda_{k^{\prime} n^{\prime} m^{\prime}}^{k n m} \neq 0$ for at most two systems $\left(k^{\prime}, n^{\prime}, m^{\prime}\right)$ such that $k^{\prime}+n^{\prime}+m^{\prime}-k-n-m$ is odd. So, $b_{1} \ldots b_{r} b_{r+1}=\sum_{k^{\prime}, n^{\prime}, m^{\prime}} \lambda_{k^{\prime} n^{\prime} m^{\prime}}^{\prime} a_{k^{\prime} n^{\prime} m^{\prime}}$, where

$$
\lambda_{k^{\prime} n^{\prime} m^{\prime}}^{\prime}=\sum_{k, n, m} \lambda_{k n m} \lambda_{k^{\prime} n^{\prime} m^{\prime}}^{k n m}
$$

and the summation is over such systems $(k, n, m)$ that $k^{\prime}+n^{\prime}+m^{\prime}-k-n-m$ is odd. Suppose now that $\lambda_{k^{\prime} n^{\prime} m^{\prime}}^{\prime} \neq 0$ for some $k^{\prime}, n^{\prime}, m^{\prime}$. It means that in (5.3) there is a nonzero ingredient, i.e. there is a system $(k, n, m)$ such that $k^{\prime}+n^{\prime}+m^{\prime}-k-n-m$ is odd and $\lambda_{k n m} \neq 0 . k+n+m-r$ is even by the inductive assumption. Then, $k^{\prime}+n^{\prime}+m^{\prime}-(r+1)$ is even too.

Let us fix some important elements in $\mathcal{O}_{2}^{\alpha}$ (see [24]):

$$
\theta=\psi_{1} \psi_{1} \psi_{1}^{*} \psi_{1}^{*}+\psi_{2} \psi_{2} \psi_{2}^{*} \psi_{2}^{*}+q \psi_{2} \psi_{1} \psi_{2}^{*} \psi_{1}^{*}+q \psi_{1} \psi_{2} \psi_{1}^{*} \psi_{2}^{*}+\left(1-q^{2}\right) \psi_{2} \psi_{1} \psi_{1}^{*} \psi_{2}^{*},
$$

$$
S=\left(1+q^{2}\right)^{-\frac{1}{2}}\left(\psi_{1} \psi_{2}-q \psi_{2} \psi_{1}\right)
$$


Remark 5.2. The selfadjoint element $\theta \in H^{2} H^{* 2}$ fulfills the following relations:

H1) $\sigma^{n}(\theta) \sigma^{n+1}(\theta) \sigma^{n}(\theta)=\sigma^{n+1}(\theta) \sigma^{n}(\theta) \sigma^{n+1}(\theta)$ for $n \in \mathbf{N}_{0}$,

H2) $\sigma^{n}(\theta) \sigma^{m}(\theta)=\sigma^{m}(\theta) \sigma^{n}(\theta)$ for $n, m \in \mathbf{N}_{0}$ such that $|n-m|>1$,

H3) $\theta^{2}=\left(1-q^{2}\right) \theta+q^{2} I$.

Moreover, it is known ([24]) that

H4) for every $n \geq 2$ the $C^{*}$-algebra $\mathcal{O}_{2}^{\alpha} \cap H^{n} H^{* n}$ is generated by elements $\sigma^{m}(\theta)$, $m=0, \ldots, n-2$.

For $q=1$ the element $\theta$ is just the flip operator on $H^{2} H^{* 2} \cong M_{2}(\mathbf{C}) \otimes M_{2}(\mathbf{C})$. Moreover, if $1 \leq m \leq n-1$ then $\sigma^{m-1}(\theta)$ interchanges $m$-th and $(m+1)$-th factors in $H^{n} H^{* n} \cong M_{2}(\mathbf{C})^{\otimes n}$. Let $s_{1}, \ldots, s_{n-1} \in S_{n}$ be reflections generating the symmetric group $S_{n}$. Then the mapping $s_{m} \mapsto \sigma^{m-1}(\theta)$ can be extended to a representation of the group $S_{n}$ in the algebra $H^{n} H^{* n}$. By taking inductive limits we obtain a representation of $S_{\infty}$ on the UHF-algebra $\mathcal{O}_{2}^{0} \cong M_{2}(\mathbf{C})^{\otimes \infty}$ which is nothing but the one constructed in [17] and [16] (see also [18]). Of course this representation can be extended to a representation $\pi$ of the algebra $\mathbf{C}\left(S_{\infty}\right)$ (the inductive limit of the group algebras $\mathbf{C}\left(S_{n}\right)$ ) in the algebra $\mathcal{O}_{2}^{0}$. Thus we arrive at the permutation symmetry (see [4]) and we have $\pi\left(\mathbf{C}\left(S_{\infty}\right)\right)=\mathcal{O}_{2}^{S U(2)} \cap \mathcal{O}_{2}^{0}$.

For an arbitrary $q \in[-1,1] \backslash\{0\}$ let $g_{1}, \ldots, g_{n-1}$ be the generators of the Hecke algebra $H_{n}\left(q^{2}\right)$ (see [21]). Then due to the relations H1)-H3) from Remark 5.2 the mapping $g_{m} \mapsto \sigma^{m-1}(\theta), m=1, \ldots, n-1$, can be extended to a representation of $H_{n}\left(q^{2}\right)$ in $H^{n} H^{* n}$. Moreover, the similar inductive limits argument leads to a representation $\pi_{q}$ of $H\left(q^{2}\right)$ in $\mathcal{O}_{2}^{0}$.

Proposition 5.3. 1. The element $S \in H^{2}$ defined in (5.5) is an isometry and $S S^{*} \in H^{2} H^{* 2}$ is a spectral projection of $\theta$.

Moreover, $S$ fulfills the following relations:

2. $S^{*} \sigma(S)=-\left(q+q^{-1}\right)^{-1} I$,

3. $\sigma^{n}\left(S S^{*}\right) \sigma^{m}\left(S S^{*}\right)=\sigma^{m}\left(S S^{*}\right) \sigma^{n}\left(S S^{*}\right)$ for $n, m \in \boldsymbol{N}_{0}$ such that $|n-m|>1$,

4. $\sigma^{n}\left(S S^{*}\right) \sigma^{m}\left(S S^{*}\right) \sigma^{n}\left(S S^{*}\right)=\left(q+q^{-1}\right)^{-2} \sigma^{n}\left(S S^{*}\right)$ for $n, m \in \boldsymbol{N}_{0}$ such that $|n-m|=1$.

Proof. 1. $\theta=I-\left(1+q^{2}\right) S S^{*}=\left(I-S S^{*}\right)-q^{2} S S^{*}$. The other points are consequences of simple calculations.

Remark 5.4. 5.3.3 and 5.3.4 show that the family $\left\{\sigma^{l}\left(S S^{*}\right): l \in \mathbf{N}_{0}\right\}$ can be considered as the system of Jones' projections (see [12]).

Theorem 5.5. $\mathcal{O}_{2}^{\alpha}$ is the smallest $C^{*}$-subalgebra of $\mathcal{O}_{2}$ which contains $S$ and is stable under the canonical endomorphism $\rho$.

Proof. Let $C^{*}(S, \rho)$ denote the smallest $C^{*}$-algebra containing $S$ and invariant under $\rho$. It is obvious that $C^{*}(S, \rho) \subset \mathcal{O}_{2}^{\alpha}$ (cf. Lemma 3.5). In order to prove the converse inclusion, first we will show that $\mathcal{O}_{2}^{\alpha} \cap \mathcal{P}_{2} \subset C^{*}(S, \rho)$. By Proposition 4.3 it suffices to prove that $\mathcal{O}_{2}^{\alpha} \cap \mathcal{P}_{2}^{k} \subset C^{*}(S, \rho)$ for every $k \in \mathbf{Z}$. Consider the case $k=0 . \quad \mathcal{O}_{2}^{\alpha} \cap \mathcal{P}_{2}^{0}=\bigcup_{n} \mathcal{O}_{2}^{\alpha} \cap H^{n} H^{* n}$ and from Remark 5.2.H4) we see that $\mathcal{O}_{2}^{\alpha} \cap \mathcal{P}_{2}^{0}$ is generated by $\sigma^{m}(\theta), m \in \mathbf{N}_{0}$. Proposition 5.3 implies that $\theta \in C^{*}(S, \rho)$, so $\mathcal{O}_{2}^{\alpha} \cap \mathcal{P}_{2}^{0} \subset C^{*}(S, \rho)$. Next, let $X \in \mathcal{O}_{2}^{\alpha} \cap \mathcal{P}_{2}^{2 l}$ for some $l \in \mathbf{Z}$. By taking $X^{*}$ if necessary, we can assume that $l \geq 0$. Then $X S^{* l} \in \mathcal{O}_{2}^{\alpha} \cap \mathcal{P}_{2}^{0} \subset C^{*}(S, \rho)$ and $X=X S^{* l} S^{l} \in C^{*}(S, \rho)$. It remains to show that $\mathcal{O}_{2}^{\alpha} \cap \mathcal{P}_{2}^{2 l+1}=\{0\}$ for every $l \in \mathbf{Z}$. Proposition 4.4 yields

$$
\mathcal{O}_{2}^{\alpha} \cap \mathcal{P}_{2}^{2 l+1}=E_{\alpha}\left(\mathcal{P}_{2}^{2 l+1}\right) .
$$


Let $X$ be an element of $\mathcal{P}_{2}^{2 l+1}$ of the form

$$
X=\psi_{i_{1}} \ldots \psi_{i_{n}} \psi_{i_{n+1}} \ldots \psi_{i_{n+2 l+1}} \psi_{j_{n}}^{*} \ldots \psi_{j_{1}}^{*}
$$

for some $n \in \mathbf{N}$ and $i_{1}, \ldots, i_{n+2 l+1}, j_{1}, \ldots, j_{n}=1,2$. Then

$$
\begin{aligned}
E_{\alpha}(X) & =\sum_{\substack{p_{1}, \ldots, p_{n+2 l+1} \\
r_{1}, \ldots, r_{n}}} h\left(u_{p_{1} i_{1}} \ldots u_{p_{n+2 l+1} i_{n+2 l+1}} u_{r_{n} j_{n}}^{*} \ldots u_{r_{1} j_{1}}^{*}\right) \\
& \times \quad \psi_{p_{1}} \ldots \psi_{p_{n+2 l+1}} \psi_{r_{n}}^{*} \ldots \psi_{r_{1}}^{*}
\end{aligned}
$$

where matrix elements $u_{i j}$ are given by (5.2). Thus, Lemma 5.1 implies that the element $u_{p_{1} i_{1}} \ldots u_{p_{n+2 l+1} i_{n+2 l+1}} u_{r_{n} j_{n}}^{*} \ldots u_{r_{1} j_{1}}^{*}$ is a linear combination of elements $a_{k n m}$ such that $k+n+m$ is odd. But $h\left(a_{k n m}\right) \neq 0$ if and only if $k=$ 0 and $n=m([23])$, so $h\left(u_{p_{1} i_{1}} \ldots u_{p_{n+2 l+1} i_{n+2 l+1}} u_{r_{n} j_{n}}^{*} \ldots u_{r_{1} j_{1}}^{*}\right)=0$ for every $i_{1}, \ldots, i_{n+2 l+1}, j_{1}, \ldots, j_{n}, p_{1}, \ldots, p_{n+2 l+1}, r_{1}, \ldots, r_{n}$. Therefore, $E_{\alpha}(X)=0 . \mathcal{P}_{2}^{2 l+1}$ is spanned by elements of the form (5.7) (see (4.1)), so $E_{\alpha}(X)=0$ for every $X \in \mathcal{P}_{2}^{2 l+1}$ and by (5.6) $\mathcal{O}_{2}^{\alpha} \cap \mathcal{P}_{2}^{2 l+1}=\{0\}$. Secondly, we know that $\mathcal{O}_{2}^{\alpha} \cap \mathcal{P}_{2}$ is dense in $\mathcal{O}_{2}^{\alpha}$ (see Lemma 6 of [12]). Therefore it is clear that $\mathcal{O}_{2}^{\alpha} \subset C^{*}(S, \rho)$.

Lemma 4.6 and the above theorem lead to

Lemma 5.6. The subalgebra $\mathcal{O}_{2}^{\alpha}$ has the trivial relative commutant in $\mathcal{O}_{2}$, i.e. $\left(\mathcal{O}_{2}^{\alpha}\right)^{\prime} \cap \mathcal{O}_{2}=C I$.

Consequently, Theorem 3.6 and Lemma 5.6 give

Corollary 5.7. For every $X \in \mathcal{O}_{2}^{\alpha}$ the following conditions are equivalent:

i) $X \in \operatorname{Mor}\left(\alpha^{n}, \alpha^{m}\right)$,

ii) $X \sigma^{n}(Y)=\sigma^{m}(Y) X$, for $Y \in \mathcal{O}_{2}^{\alpha}$.

We conclude this section with a discussion of the structure of the fixed point algebra $\mathcal{O}_{2}^{\alpha}$. To this end let us recall that the authors of [12] have considered the twisted unitary group ${ }_{q} U(2)$ and its action $\alpha^{\prime}$ on $\mathcal{O}_{2}$ is. They have proved that the fixed point algebra $\mathcal{O}_{2}^{\alpha^{\prime}}$ of this action is exactly $\mathcal{O}_{2}^{\alpha} \cap \mathcal{O}_{2}^{0}$.

Let $\tau(A)=S A S^{*}, A \in \mathcal{O}_{2}^{\alpha^{\prime}}$, be the endomorphism of $\mathcal{O}_{2}^{\alpha^{\prime}}$ induced by the isometry $S$. From the proof of Theorem 5.3 we can observe that every element $A \in \mathcal{O}_{2}^{\alpha} \cap \mathcal{O}_{2}^{2 l}$ is of the form

$$
A= \begin{cases}B S^{l}, & \text { if } l \geq 0 \\ S^{* l} B, & \text { if } l<0\end{cases}
$$

where $B \in \mathcal{O}_{2}^{\alpha^{\prime}}$. Then, $\mathcal{O}_{2}^{\alpha}$ has the structure of crossed product of $\mathcal{O}_{2}^{\alpha^{\prime}}$ by the endomorphism $\tau$ (see [15]). Indeed, we have the following universal property ( $c f$. Lemma 3.8 of [5])

Proposition 5.8. Let $\varphi_{0}: \mathcal{O}_{2}^{\alpha^{\prime}} \rightarrow \mathcal{B}$ be a morphism of unital $C^{*}$-algebras and $R \in \mathcal{B}$ be an isometry of $\mathcal{B}$ such that $R \varphi_{0}(A) R^{*}=\varphi_{0}(\tau(A))$, for $A \in \mathcal{O}_{2}^{\alpha^{\prime}}$. Then $\varphi_{0}$ extends uniquely to a morphism $\varphi: \mathcal{O}_{2}^{\alpha} \rightarrow \mathcal{B}$ with $\varphi(S)=R$.

Proof. It is the simple consequence of (5.8). 


\section{REFERENCES}

[1] Araki H., Carey A.L., Evans D.E., $O n O_{n+1}$, J. Operator Theory 12 (1984), 247-264 MR 86g:46082

[2] Bratteli O., Evans D.E., Derivations tangential to compact groups: the non-abelian case, Proc. London Math. Soc. 52 (1986), 369-384 MR 87f:46123

[3] Cuntz J., Simple $C^{*}$-algebras generated by isometries, Comm. Math. Phys. 57 (1977), 173185 MR 57:7189

[4] Doplicher S., Roberts J.E., Duals of Compact Lie Groups Realized in the Cuntz Algebras and Their Actions on $C^{*}$-Algebras, J. Funct. Anal. 74 (1987), 96-120 MR 89a:22011

[5] Doplicher S., Roberts J.E., Compact group actions on $C^{*}$-algebras, J. Operator Theory 19 (1988), 283-305 MR 90f:46104

[6] Doplicher S., Roberts J.E., A new duality theory for compact groups, Invent. Math. 98 (1989), 157-218 MR 90k:22005

[7] Doplicher S., Roberts J.E., Why There is a Field Algebra with a Compact Gauge Group Describing the Superselection Structure in Particle Physics, Comm. Math. Phys. 131 (1990), 51-107 MR 91k:81082

[8] Fannes M., Nachtergaele B., Werner R.F., Quantum Spin Chains with Quantum Group Symmetry, Comm. Math. Phys. 174 (1996), 477-507 CMP 96:07

[9] Fredenhagen K., Rehren K.-H., Schroer B., Superselection Sectors with Braid Group Statistics and Exchange Algebras I, Comm. Math. Phys. 125 (1989), 201-226 MR 91c:81047

[10] Fredenhagen K., Rehren K.-H., Schroer B., Superselection Sectors with Braid Group Statistics and Exchange Algebras II, Rev. Math. Phys. - Special Issue (1992), 113-157 MR 94g:81119

[11] Haag R., Local Quantum Physics, Springer-Verlag, Berlin Heidelberg 1992 MR 94d:81001

[12] Konishi Y., Nagisa M., Watatani Y., Some remarks on actions of compact matrix quantum groups on $C^{*}$-algebras, Pacific J. Math. 153 (1992), 119-127 MR 93c:46121

[13] Mack G., Schomerus V., Conformal Field Algebras with Quantum Symmetry from the Theory of Superselection Sectors, Comm. Math. Phys. 134 (1990), 139-196 MR 92i:81298

[14] Mack G., Schomerus V., Quasi Hopf quantum symmetry in quantum theory, Nucl. Phys. B 370 (1992), 185-230 MR 93c:81081

[15] Paschke W.L., The crossed product of a $C^{*}$-algebra by an endomorphism, Proc. Amer. Math. Soc. 80 (1980), 113-118 MR 81m:46081

[16] Powers R.T., Price G., Derivations Vanishing on $S(\infty)$, Comm. Math. Phys. 84 (1982), 439-447 MR 83k:46056

[17] Price G., Extremal Traces on Some Group-Invariant $C^{*}$-Algebras, J. Funct. Anal. 49 (1982), 145-151 MR 84e:46075

[18] Størmer E., Symmetric States of Infinite Tensor Products of $C^{*}$-algebras, J. Funct. Anal. 3 (1969), 48-68 MR 39:3327

[19] Wang S., Free Products of Compact Quantum Groups, Comm. Math. Phys. 167 (1995), 671-692 MR 95k:46104

[20] Wang S., Tensor products and crossed products of compact quantum groups, Proc. London Math. Soc. 71 (1995), 695-720 MR 96i:46074

[21] Wenzl H., Hecke algebras of type $A_{n}$ and subfactors, Invent. Math. 92 (1988), 349-383 MR 90b: 46118

[22] Woronowicz S.L., Twisted SU(2) group. An example of a non-commutative differential calculus, R.I.M.S. Publ. Kyoto University 23 (1987), 117-181 MR 88h:46130

[23] Woronowicz S.L., Compact Matrix Pseudogroups, Comm. Math. Phys. 111 (1987), 613-665 MR 88m:46079

[24] Woronowicz S.L., Tannaka-Krein duality for compact matrix pseudogroups. Twisted SU(N) groups, Invent. Math. 93 (1988), 35-76 MR 90e:22033

[25] Woronowicz S.L., Compact quantum groups, preprint, University of Warsaw.

Institute of Mathematics, Gdańsk University, Wita Stwosza 57, 80-952 Gdańsk, PoLAND

E-mail address: matmm@univ.gda.pl 